\title{
A Head-Driven Approach to Incremental and Parallel Generation of Syntactic Structures
}

\author{
Günter Neumann \\ Institute for Computational Linguistics \\ University of Saarbrücken \\ Im Stadtwald 15, Bau 17 \\ 6600 Saarbrücken 11, FRG \\ neumann@sbuvax.campus.uni-sb.de
}

\author{
Wolfgang Finkler \\ German Research Center for \\ Artificial Intelligence \\ Stuhlsatzenhausweg 3 \\ 6600 Saarbrüicken 11 , FRG \\ finkler@dfki.uni-sb.de
}

\begin{abstract}
This paper describes the construction of syntactic structures within an incremental multi-level and parallel generation system. Incremental and parallel generation imposes special requirements upon syntactic description and processing. A head-driven grammar represented in a unification-based formalism is introduced which satisfies these demands. Furthermore the basic mechanisms for the parallel processing of syntactic segments are presented.
\end{abstract}

\section{Introduction}

Incremental generation (i.e. immediate verbalization of the parts of a stepwise computed conceptual structure - often called "message") is an important and efficient property of human language use ([DeSmedt\&Kempen87], [Levelt89]). There are particular situations of communication (e.g. simultaneous descriptions of ongoing events) where incremental generation is necessary in order to ensure that new information can be verbalized in due time. As [DeSmedt\& Kempen87] mentioned, incremental generation can be viewed as a parallel process: While the linguistic module (or "how-to-say" component) of an incremental generator is processing partial conceptual structures which are previously computed from the conceptual module (or "what-to-say" component) the latter can run

\footnotetext{
$1_{\text {Major parts of the work presented in this paper were }}$ developed during the master's thesis of the authors. This work was supported by the German Science Foundation (DFG) in its Special Collaborative Program on AI and Knowledge-Based Systems (SFB 314), project XTRA.

Thanks to Gregor Erbach, Norbert Reithinger and Harald Trost for their helpful comments on earlier versions of the paper.
}

simultaneously and add more conceptual elements. In [Finkler\&Neumann89] we show that it is possible to refine this parallelism: Every conceptual or linguistic segment can be viewed as an active unit which tries to verbalize itself as fast and as independently as possible. If the translation of a segment is not possible because of unspecified but required information, it is necessary to request missing information in order not to jeopardize the fast mapping. As a consequence, the linguistic module provides feedback for the selection of what to say next by the conceptual module (cf. [Hovy87], [Reithinger88]).

Incremental generation imposes special requirements upon syntactic description and processing (cf. [Kempen87]). Until now only a few approaches to syntax have been developed which consider explicitly the requirements of incremental construction of sentences, namely that of [Kempen87] and [DeSmedt\& Kempen88]. But those do not provide for a bidirectional flow of control between conceptual and linguistic module.

In this paper we describe the principles of representation and processing of syntactic knowledge in the natural language generation system POPEL-HOW, which for the first time combines explicitly incremental multi-level generation with parallelism and feedback. The syntactic knowledge is declaratively represented by a unification-based head-driven grammar that is linguistically based on dependency grammar.

\section{Requirements upon the Syntactic Level}

The following aspects concerning the representation of syntactic knowledge and the basic mechanism have to be regarded in an incremental multi-level and parallel model:

1. The grammar should be lexically based. The lexicon is assumed to be the "essential 
mediator" between conceptualization and grammatical encoding (cf. [Levelt89]). During incremental generation it is not plausible to assume that the syntactic structure is built up "from phrases to lexical elements" starting with a root node $S$.

2. The grammar should support vertical rather than horizontal orientation [Kempen87]. The rules should emphasize the growing of syntactic structures by individual branches (icieally, only by one branch, but see 4.1). One should avoid that, because of adding a new segment, unnecessary constraints are simultaneously added for sister segments.

3. Syntactic segments should be expanded in the following three ways [Kemper87]: upward (a new segment $B$ becomes the root node of a previous segment $\mathrm{A})$, downward (B becomes a daughter node of $\mathrm{A}$ ) and insertion (B becomes the daughter node of $A$ which already has a daughter and this daughter becomes the daughter of $B)^{2}$.

4. During incremental generation one should not assume that the chronological order in which syntactic segments are attached corresponds to the linear order of the resulting utterance. Hence one should separate knowitedge concerning immediate dominance and linear precedence [Kempen87]. Especially during the parallel processing of languages with a relatively free word order (e.g., German), one should avoid building tip unnecessary syntactic paraphrases resulting from ordering variations.

5. When the whole syntactic structure of an utterance is buili up in a parallel fashion, it should be possible to decide for every partial structure whether it is locally complete. In a head-driven grammar this is possible if segments are based on head elements. Thus only the information that is necessary for the inflection and linearization of the head is considered (see 4.2).

6. During spontaneous speech it may happen that already existing structures need to be modified because of new information that can only be considered by replacing cld one (which means "reformulation"). Because of the dynamic behaviour of an incremental multilevel and parallel system, syntactic structures should not only cooperate but also compete during generation (see 4.3).

The basic demands 2., 3., 4. are put forward by Kempen's framework for syntactic tree formation [Kempen87]. They serve as a

\footnotetext{
In our formalism we need only downward and upward expansion because of the nature of the syntactic structures.
}

theoretical basis for the development of the syntactic formalism proposed in this paper. The other aspects are explicitly mentioned because they are important for incremental multi-level and parallel generation. Before the formalism and the basic operations are described in more detail we brierly introduce POPEL_HOW, the generator in which the formalism is embedded.

\section{Overview of POPEL-HOW}

POPEL-HOW [Firkler\&Neumann89] is the how-lo-say component of the natural language generation system POPEL [Reithinger88]. The main features of POPEL. HOW are:

Incremental generation: POPEL-WHAT (the what-to-say component) immediately passes segments of the conceptual structure as input to POPEL-HOW when they are considered relevant for the contents to be produced. POPEL-HOW tries to build up the corresponding semantic and syntactic segments immediately, too, in order to utter the input segments as fast as possible, i.e. POPEL-HOW generates structures at each level in an incremental way.

Feedback: It is possible that new conceptual segments cannot be uttered directly because they lack necessary linguistic information. In this case POPEL-HOW is able to interact with POPEL-WHAT to demand the missing information. The bidirectional flow of control has two main advantages: Firstly, the determination of the contents can be done on the basis of conceptual considerations only. Therefore, POPEL-HOW is flexible enough to handle underspecified input ${ }^{3}$. Secondly, POPEL-WHAT has to regard feedback from POPEL-HOW during the computation of the further selection process. This means, an incremental system like POPEL can model the influence of linguistical restrictions on the process that determines what to say next (cf. [Hovy87], [Reithinger88]).

Reformulations: The addition of new conceptual segments could result in constructing semantic or syntactic segments that stand in competition with previously computed segments. POPEL-HOW is able to handle such reformulations.

Unified parallel processing: Every segment at each level is conceived as an active and independent process. This view is the foundation of the parallel and incremental generation with feedback at each level of

\footnotetext{
${ }^{3}$ [Ward88] show's that most generation systems lack this ability so that their inputs have been tailored to determine a good sentence.
} 
POPEL-HOW. The processing approach is uniform since every process (either conceptual, semantic or syntactic) runs the same basic program and processes its segment in the same way.

\section{The Design of the Syntactic Level}

The syrtactic level in POPEL-HOW is divided into two sublevels: the dependency-based level (DBS-level) and the level of inflected and linearized structures (ILS-level). At the DBSlevel the syntactic structure is constructed in an incremental and parallel way. At the ILS-level there exists one process which performs inflection and linearization for every incoming segment of the DBS-level.

\subsection{Representation}

The central knowledge source of both sublevels is POPELGRAM, a unification-based head driven grammar. The grammar is declaratively expressed in a modified version of the PATR formalism (cf. [Shieber85]). PATR is modified in the sense that the representation and derivation devices are separated. To use it for generation, the parser (i.e. the derivation device) is replaced by operations suitable for incremental and parallel generation ${ }^{4}$.

POPELGRAM is divided into immediate dominance (ID) and linear precedence (LP) structures 5 . In order to allow for vertical crientation, the ID-structures are furthermore divided into those that describe basic syntactic segments and others that describe the relationship between complex structures. Basic segments are phrases that consist of a (nonempty) set of constituents. At least one constituent must have a lexical category. The central point of view for the description of basic segments is that the dependency relation is defined for the constituents of a phrase: One constituent that must have a lexical category is denoted as the head of a phrase. All other constituents are denoted as complements that are immediately dependent on the head. The dependency relation of the constituents of a phrase is expressed as a feature description named STRUCT which belongs to the phrasal category. E. g., fig. 1 shows the feature set of the basic segment for constructing a sentence

\footnotetext{
${ }^{4}$ This is in contrast to [Shicber et al.89] where the same operations are used for both analysis and generation.

5 In this paper we only consider the ID-structures in more detail.
}

phrase where the head governs two complements ${ }^{6}$ :

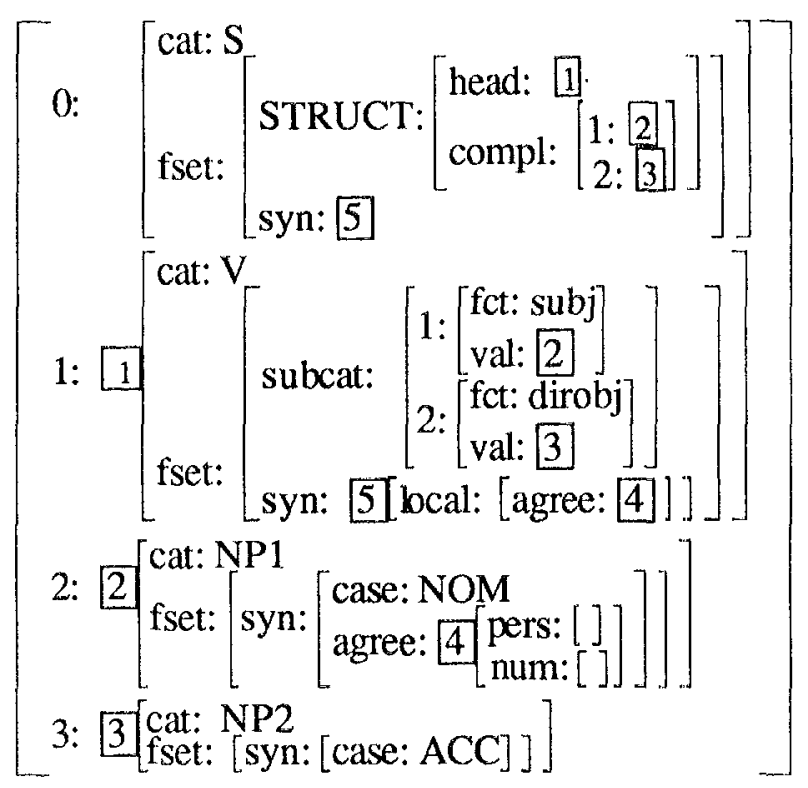

Fig. 1

The head element is the central element of the phrase and determines the characteristic properties of the whole phrase that is defined as the projection of its head ${ }^{7}$. The particular quality of ID-structures of POPELGRAM makes it possible to interpret such structures as theoretically based on dependency grammar (cf. [Kunze75], [Hellwig86]). Basic segments can also be defined as abstract descriptions of certain classes of lexical elements which have the same category and valence (or subcategorization) restrictions. The abstract description of lexical information (in the sense of dependency grammar) is the foundation of our lexically based grammar. We assume that this view supports incremental and parallel generation.

Obviously, basic segments build up syntactic constraints for their complements. Although this seems to emphasize horizontal orientation, these constraints are not redundant and therefore do not violate the view of vertical orientation. A basic segment must access the information that is necessary to build up a

\footnotetext{
${ }^{6}$ The integer attributes correspond to the order of the elements in the corresponding ID-rule

$\mathrm{S} \Rightarrow \mathrm{V}, \mathrm{NP1}, \mathrm{NP2}$. Note: The order of constituents is not assumed to be the order of the corresponding surface string.

${ }^{7}$ If complements of basic segments are defined as phrases, then the head elements of the complements are immediately dependent on the head element of the basic segment, because of the projection principle. Hence, complex syntactic structures are compositionally viewed as hierarchical head and modifier structures.
} 
minimal syntactically well-formed partial utterance. If the dependencies between a head element and its modifiers are strong as in the case of complements, then this information has to be formulated in an incremental grammar, too $^{8}$.

Adjuncts are not necessary for building minimal well-formed structures. Therefore they are not part of basic segments. The combination of basic segments and adjuncts is expressed by means of complex segments. Those IDstructures are applied only when the corresponding basic segment already exists.

\subsection{Incremental and Parallel Processing}

ID-structures are processed at the DBS-level. At this level basic segments are considered as independent and active units. In order to realize this every basic segment is associated with its own active process (or object) whereby the structure of an object reflects the underlying dependency relation of the segment. For the feature set of fig. 1 this can graphically be represented as follows:

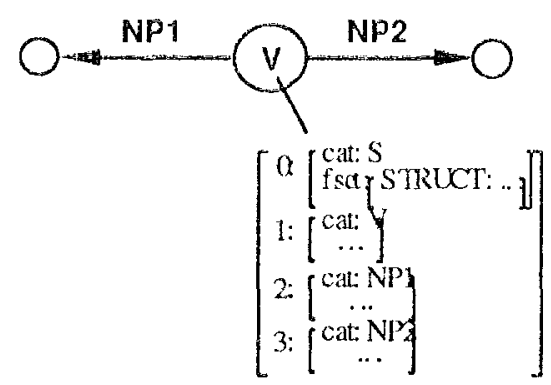

Fig. 2

(v) is denoted as the body and the directed labeled slots $\mathrm{O} \longrightarrow \mathrm{NP1}$ and $\stackrel{\mathrm{NP2}}{-}-\mathrm{O}$ as the context of the process. The names of the body and the slot correspond to the feature sets of the associated basic segment. Every labeled slot serves as a communication link to a process which represents the basic segment of the corresponding slot's complement. The topology of the network of active processes xepresents the corresponding dependency-based tree structure.

Objects at the DBS-level are created during the evaluation of local transition rules. These

\footnotetext{
${ }^{8}$ This point of view is in contrast to [Kempen87]. In his framework, all syntactic segments are defined as node-arc-node structures because segments have to branch only by one element at a time.
}

rules describe the relation between semantic and syntactic knowledge in a distributed way [Finkler\&Neumann89]. They are local because every rule describes the mapping of semantic segments (represented as concepts of a network-like formalism) to syntactic segments of the ID-part of POPELGRAM. In principle this local mapping is case-frame based: A semantic head (e.g., a predicate) and its deep cases (e.g., agent or benefactive) are related to a corresponding syntactic head (e.g. a verb) and its syntactic frame (e.g., subject or direct object constituents). During the mapping lexical material which is determined through the choice of words ${ }^{9}$ directs and restricts the choice of possible syntactic structures.

In order to construct syntactic structures in an incremental and parallel way, every DBSobject has to solve the following two central subtasks:

a. building up connections to other objects

b. mapping itself as fast as possible into the next level

In order to solve task a., every new created DBS-object tries to integrate itself into the topology of the already existing objects. Thereby the following cases have to be distinguished:

1. The new object is taken up into the context of an object. Syntactically, this means that the new object represents an immediately dependent constituent. The existing structure is expanded downward (see fig. 3).

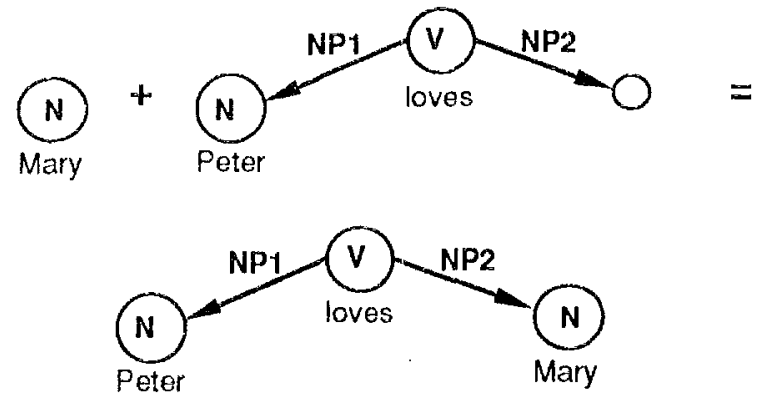

Fig, 3:

The new active object is the left one.

\footnotetext{
${ }^{9}$ The choice of words is performed in two steps in POPEL-HOW. While conceptual segments are translated into semantic segments, the content words - c.g. verbs and nouns - are selected. The selection of function words - e.g. prepositions which depend on the meaning of the verb - is performed during the mapping between the semantic level and the DBS-level.
} 
2. The new object takes up (or binds) an already existing object. The existing structure is expanded upward (see fig. 4).

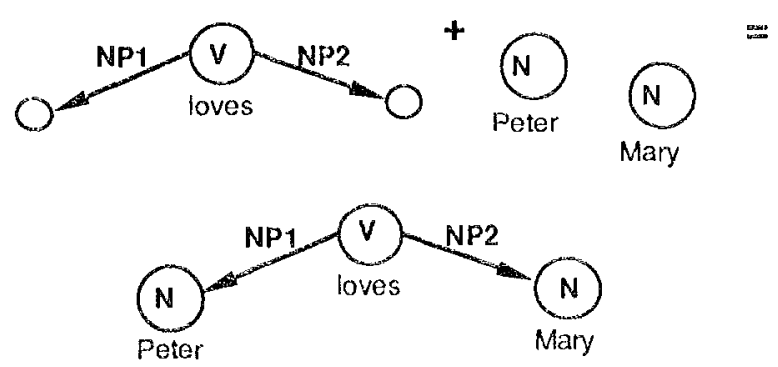

Fig. 4:

In this example the new verbal object binds two existing nouns.

3. The new object describes a reformulation of the basic segment of an already existing object. This object must be replaced with the new one (see fig. 5).

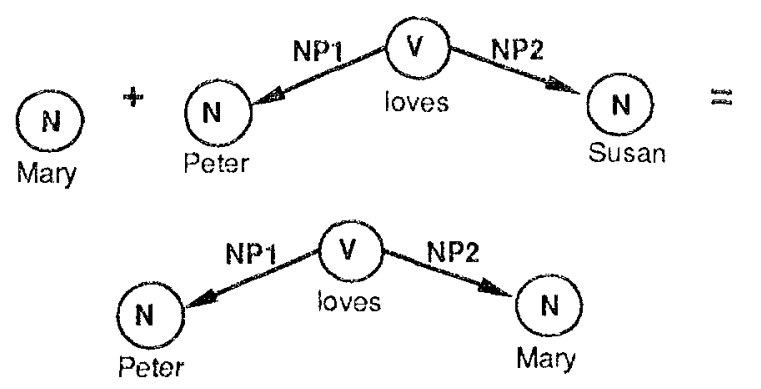

Fig. 5:

This is actually uttered as:

"Peter loves Susan ... uh ... Mary."

While new communication links are built up, participating objects exchange their syntactic features ${ }^{10}$. An adjunct is added to an object by augmenting the object's context with a new labeled slot. When the slot is being filled with an object, its associated feature set and the basic segment are combined to yield a complex segment that represents the new segment of the augmented object.

Every DBS-object tries to map itself into the next level as fast as possible (subtask b.) in

\footnotetext{
10 This is performed with the help of a search algorithm using the ID-structures of POPELGRAM. The IDstructures implicitly represent the search space. To constraint the space, heuristic information in form of partial feature descriptions is used. This search operation serves as the basis for other operations, too, e.g. for unifying lexical elements with basic segments.
}

order to facilitate spontaneous speech. The feature set of the head of the associated segment is checked to see if it contains sufficient information for inflecting and linearizing it. For every segment this information has to be specified under the head's feature local. Actual values come either from the lexicon, e.g., gender for nouns, from dependent elements by means of structure sharing, e.g., person and number for a verb from the subject constituent or from a govering constituent, e.g., case for dependent nouns of a verb.

When all subfeatures of the local feature have values, i.e. a value other than the empty feature set, then the segment is said to be locally complete. The reason for the segment of a DBS-object not to be locally complete is that DBS-objects that share values with the local feature set are either not yet created or not yet locally complete themselves (e.g., a verb is locally incomplete when a phrase that has to fill the subject role is not yet created). In the first case, the missing objects are requested (using the object's context) from that object of the semantic level above that has created the requesting object. In the second case, DBSobjects that are already connected to the underspecified object are invited to determine the missing values.

\subsection{Inflection, Linearization and Reformulation}

Segments from the DBS-level that are locally complete are inflected and linearized at the ILSlevel in POPEL.HOW. The inflection is performed by means of the package MORPHIX [Finkler\&Neumann88], which handles most of the inflection phenomena of German. The order of segments in an utterance is syntactically constrained by the LP-part of POPELGRAM. It is assumed that the order of activation of conceptual segments (which is determined using pragmatical knowledge (cf. [Reithinger88])) should be maintained if it is syntactically wellformed; otherwise the segments are reordered by means of relevant $L P$-structures. Therefore, the order of the input segments affects the variations of the linear order in the resulting utterance, which makes POPEL-HOW more flexible (e.g. by deciding whether to realize an active or passive form). But the order of the input segments can affect lexical choice, too. For example, if the modifier denoting the vehicle (e.g., "airplane") for a conceptual segment of type "commute-bypublic-transportation" is known in due time the more restricted verb "to fly" is chosen instead of the more general verb "to go". Otherwise, a 
prepositional phrase realizing the modifier must be verbalized explicitly (e.g., "to go by airplane").

It is possible that because of the incremental composition of large structures across several levels new conceptual segments lead to the reformulation of structures at successive levels (cf. [DeSmedt\&Kempen88]) as indicated in the following sample utterances:

"Mary is going ... uh ... Mary and Peter are going to school."

"Eric goes ... uh ... flies to the USA."

In POPEL HOW the reformulation of a segment is represented by an object that is in competition to the corresponding already existing object (e.g., the first utterance where the new conceptual segment "Peter" leads to a syntactic segment "Mary and Peter" which is in competition with the noun phrase "Mary"). In order to integrate such new objects the connections to an object which is to be replaced must be broken up and reconnected to the new object (see also fig. 5). Of course, the replacement must be performed for the relevant associated segments, too. For syntactic segments we have developed an operation which allows the replacement of parts of a given feature set. In principle this is realized by resetting the corresponding partial feature set (i.e., relevant features get the empty value) and subsequently unifying it with the new information.

\section{Conclusion}

This paper has demonstrated the representation and processing of syntactic knowledge within the generation system POPEL-HOW which for the first time combines explicitly incremental sentence production with parallelism and feedback. Such a generation model imposes special requirements on syntactic representation and processing. A unification-based head-driven grammar, linguistically based on dependency grammar, is introduced that satisfies these demands. Furthermore the basic mechanisms for incremental and parallel generation have been presented.

The whole generation system is implemented in Commonlisp on a Symbolics 3640 lispmachine by simulated parallelism using the process-facilities and the scheduler. It also runs in Kyoto Commonlisp and CLOS (using a selfwritten scheduler). The system is fully integrated in the natural language access system XTRA (cf. [Allgayer et al.89]).

\section{References}

[Allgayer et al.89] J. Allgayer, R. JansenWinkeln, C. Reddig and N. Reithinger. Bidirectional use of knowledge in the multi-modal NL access system XTRA: In: Proccedings of the $11 \mathrm{th}$ IJCAI, pp. 1492 - 1497, Detroit, Michigan USA, 1989.

[DeSmedt\&Kempen87] K. DeSmedt and $G$. Kempen. Incremental Sentence Production, SelfCorrection and Coordination. In: G. Kempen (cd.), Natural Language Generation: New Results in Artificial Intelligence, Psychology and Linguistics, pp. 365-376, Dordrecht: Martinus Nijhoff, 1987.

[DeSmedt\&Kempen88] K. DeSmedt and G. Kempen. The Representation of Grammatical Knowledge in a Model for Incremental Sentence Generation. Paper presented at the 4th IWG, Santa Catalina Island, 71988.

[Finkler\&Neumann88] W. Finkler and G. Neumann. MORPIIIX: A Fast Realization of a Classification-Based Approach to Morphology. In: Proceedings of the WWWS, Springer, Berlin, 1988.

[Finkler\&Neumann89] W. Finkler and $G$. Neumann. POPEL-HOW: A Distributed Parallel Model for Incremental Natural Language Production with Feedback. In: Proceedings of the 11 th IJCAI, pp. 1518 - 1523, Detroit, Michigan USA, 1989.

[Hellwig86] P. Hellwig. Dependency Unification Grammar. In: Proceedings of the 11 th COLING, Bonn, FRG, 1986

[Hovy87] E. Hovy. Generating Naiural Language Under Pragmatic Constraints. Ph.D. thesis, Yale University, 1987.

[Kempen87] G. Kempen. A Framework for Incremental Syntactic Tree Formation. In: Procedings of the 10th IJCAI, Mailand, Italy, 1987.

[Kunze75] J. Kunze. Abhängigkeitsgrammatik. Akademie-Verlag, Berlin, 1975.

[Level189] W.J.M. Levelt. Speaking: From Intention to Articulation. Massachusetts Institute of 'Technology: The MIT Press, 1989.

[Reithinger88] N. Reithinger. POPEL: A Parallel and Incremental Natural Language Generation System. Paper presented at the 4th IWG, Santa Catalina Island, 7 1988.

[Shieber85] S.M. Shieber. An Introduction to Unification-Based Approaches to Grammar. Volume 4 of CSLI Lecture Notes, CLSI, Stanford, California, 1985.

[Shicber et al.89] S.M. Shieber, G. van Noord, R.M. Moore and F.C.P. Pereira. A Semantic HeadDriven Generation Algorithm for Unification-Based Formalisms. In: Proceedings of the 27 th $\mathrm{ACL}$, Vancouver, British Columbia, Canada, 1989.

[Ward88] N. Ward. Issues in Word Choice. In: Proceedings of the 12 th COLING, Budapest, Hungary, 1988. 